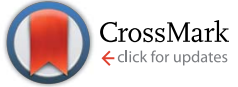

Cite this: RSC Adv., 2017, 7, 11626

Received 9th December 2016 Accepted 9th February 2017

DOI: $10.1039 / c 6 r a 27986 d$

rsc.li/rsc-advances

\section{Enhancement of textile-dyeing sludge dewaterability using a novel cationic polyacrylamide: role of cationic block structures $\uparrow$}

\author{
Li Feng, ${ }^{\text {abc }}$ Huaili Zheng, ${ }^{\text {*abc }}$ Baoyu Gao, ${ }^{d}$ Chuanliang Zhao, ${ }^{\text {abc }}$ Shixin Zhang ${ }^{\text {abc }}$ \\ and Nan Chen ${ }^{\mathrm{abc}}$
}

In this study, a novel cationic polyacrylamide (CPAM) with a microblock structure was successfully synthesized through ultrasonic-initiated template copolymerization (UTP) using allyltrimethylammonium chloride (TM) and acrylamide (AM) as monomers, and sodium polyacrylate (NaPAA) as a template. Fourier transform infrared spectroscopy (FT-IR), ${ }^{1} \mathrm{H}\left({ }^{13} \mathrm{C}\right)$ nuclear magnetic resonance spectroscopy $\left({ }^{1} \mathrm{H}\left({ }^{13} \mathrm{C}\right)\right.$ NMR), and thermogravimetric analysis (TGA) were employed to characterize the properties of the polymers. The results showed that the novel cationic microblock structure was formed in the template copolymer of TM and AM (TPTA). Besides, the copolymerization was demonstrated to follow an I zip-up (ZIP) template polymerization mechanism through the analysis of association constant $\left(M_{K}\right)$ and polymerization kinetics. The flocculation results of textile-dyeing sludge dewatering revealed that the polymer with the novel microblock structure showed an excellent flocculation performance. When the optimal conditions at $\mathrm{pH}$ of 7.0, dosage of $40 \mathrm{mg} \mathrm{L}^{-1}$ and the intrinsic viscosity of $2.3 \mathrm{dL} \mathrm{g}^{-1}$, the minimum SRF of $4.9 \times 10^{12} \mathrm{~m} \mathrm{~kg}^{-1}$ and FCMC of $72.1 \%$ were observed. During the flocculation process, the cationic microblocks in TPTA extremely enhanced the ability of charge neutralization and bridging, and contributed much to the excellent flocculation performance in textile-dyeing sludge dewatering.

\section{Introduction}

With the increase of textile-dyeing wastewater generation and wastewater treatment efficiency, the growth rate of textiledyeing sludge is going up year by year. According to the China Environment Statistical Yearbook, the amount of textile-dyeing sludge in China was up to approximately 5.38 million tons (80\% moisture content) in 2013 in China., ${ }^{\mathbf{1} 2}$ Unfortunately, this already huge production rate was predicted to further increase due to more progressively stringent wastewater effluent regulation criteria as well as the growing population. Textile sludge dewatering and reduction has become a global challenging issue for water industries and governments. ${ }^{3}$ Therefore, research on textile sludge dewatering becomes more urgent and significant.

${ }^{a}$ Key Laboratory of the Three Gorges Reservoir Region's Eco-Environment, Ministry of Education, Chongqing University, Chongqing 400045, China. E-mail: zhl6512@126. com; Fax: +8623 65120827; Tel: +862365120827

${ }^{b}$ National Centre for International Research of Low-Carbon and Green Buildings, Chongqing University, Chongqing 400045, China

${ }^{c}$ Chongqing Engineering Research Center of Water Treatment Coagulant, Chongqing 400045, China

${ }^{d}$ Shandong Key Laboratory of Water Pollution Control and Resource Reuse, School of Environmental Science and Engineering, Shandong University, Jinan 250100, China

$\dagger$ Electronic supplementary information (ESI) available. See DOI: $10.1039 /$ c6ra27986d
Currently, chemical conditioners, especially cationic polyacrylamide (CPAM), have triggered intensive interest because of their superiorities such as high efficiency, friendly-environment, facile operation and economic advantages. ${ }^{4}$ The textile sludge colloids were destabilized and agglomerated by CPAM to form large flocs through charge neutralization and bridging, which could reduce the sludge moisture content and volume. ${ }^{5}$ So far a great number of efficient flocculants have been successfully synthesized and a desirable flocculation performance has been achieved in sludge dewatering by using those flocculants. ${ }^{6-8}$ However, the CPAM synthesized with acrylamide (AM) and cationic monomer was limited to further improve and enhance its flocculation and dewatering performance by a significant drawback of the disordered and random cationic units distribution in the polymer chain. The randomly scattering cationic units could not maximize the charge neutralization ability completely. As a result, the flocculation efficiency would be discounted greatly. ${ }^{9}$

Template polymerization technology provided a new way for the research of CPAM, which could be employed to synthesize flocculant with microblock structure. ${ }^{10}$ Similar to the rule of DNA replication, the cationic monomer would be tightly adsorbed and regularly distributed along with the anionic template molecular chain through electrostatic force, and the cationic monomer would be homopolymerized to form cationic microblock structure through the radical initiation reaction. The ability of charge neutralization could be greatly enhanced 
by the novel cationic microblock structure because flocculants with these novel structures were more efficient in neutralizing counterions, thus promoting flocculation and improving the flocculation efficiency. ${ }^{\mathbf{1 1}}$ Ultrasonic-initiated copolymerization has become more and more popular because it not only tolerates lower reaction temperature, less initiator and shorter polymerization time, but also has many advantages such as its facile operation, low cost and friendly-environment. ${ }^{\mathbf{1 2 , 1 3}}$ Furthermore, it was reported that ultrasonic-template technique has been employed to synthesize novel nanoparticles. ${ }^{\mathbf{1 4}}$ Therefore, this technique was proposed to synthesize CPAM with the novel cationic microblock structure, and it was beneficial for overcoming the problems associated with traditional polymerization technology. As the common and nontoxic monomers, allyltrimethylammonium chloride (TM) and AM were chosen to construct a novel microblock structure through ultrasonic-initiated template copolymerization (UTP). In addition, the template polymerization mechanism should be deeply studied and researched to ignite fire in the domain of polymer science and similar.

This study aimed to investigate the possibility of synthesizing the flocculant with the cationic microblock structure through UTP using AM and TM as monomers, and NaPAA as template. The structural characters of polymers synthesized though UTP and non-UTP were analyzed by many advanced instruments such as FT-IR, ${ }^{1} \mathrm{H}\left({ }^{13} \mathrm{C}\right) \mathrm{NMR}$ and TGA. Besides, the association constant $M_{\mathrm{K}}$ and polymerization kinetics were researched and analyzed to further understand the polymerization mechanism. Finally, the effect of the cationic microblocks on the sludge dewatering were investigated, and the sludge dewatering performance was evaluated in terms of filter cake moisture content (FCMC), specific resistance to filtration (SRF) and the flocs settling behaviors.

\section{Materials and methods}

\subsection{Materials}

The details of the reagents used in this experiment were as follows: the monomer AM was obtained from Chongqing Lanjie
Tap Water Company (Chongqing, China). Allyltrimethylammonium chloride (TM; $98 \mathrm{wt} \%$ in water) and urea $\left[\mathrm{CO}\left(\mathrm{NH}_{2}\right)_{2}\right]$ were purchased from Ruiqi Life Science Co., Ltd. (Shanghai, China). Template sodium polyacrylate (NaPAA) was obtained from Shandong Xintai Water Treatment (Zaozhuang, China), and its molecular weight (MW) was 4200. Initiator 2,2'azobis[2-(2-imidazolin-2-yl)propane] dihydrochloride (VA-044) was sourced from Apotheker Chemical Reagent Co., Ltd. (Chendou, China). AM and TM were of technical grade, whereas the other reagents were of analytical grade. Deionized water was used throughout the experiment. Commercial flocculants CPDA and CPMA were named as CCPDA and CCPMA, respectively. CPDA was the copolymer of AM and acryloyloxyethyl trimethyl ammonium chloride (DAC), and CPMA was synthesized by AM and methacryloxyethyl trimethyl ammonium chloride (DMC). The intrinsic viscosities of CCPDA and CCPMA were all $2.3 \mathrm{dL} \mathrm{g}^{-1}$.

\subsection{Preparation of copolymers}

The ultrasonic-initiated template copolymerization process was shown in Fig. 1, and the template copolymer TPTA was prepared as follows. A certain amount of AM (67.61 mmol), TM (23.59 $\mathrm{mmol})$, urea (0.133 mmol), NaPAA $\left(23.59 \mathrm{mmol} ; n_{\mathrm{NaPAA}}: n_{\mathrm{TM}}=\right.$ $1: 1)$ and deionized water $(0.67 \mathrm{~mol})$ was added into $100 \mathrm{~mL}$ quartz jar. Then, the $\mathrm{pH}$ of the reaction solution was adjusted to about 2.0 by $0.5 \mathrm{~mol} \mathrm{~L}^{-1} \mathrm{HCl}$ and $\mathrm{NaOH}$. The mixture temperature was kept at $20^{\circ} \mathrm{C}$ and it was exposed under the ultrasonic wave radiation for $30 \mathrm{~min}$ to form a homogeneous solution. The ultrasonic wave was generated by an ultrasonicator (KQ 2200E, Kunshan Ultrasonic Instrument Co., LTD, China) and the frequency of that was $45 \mathrm{kHz}$. After that, the solution was deoxygenated through nitrogen bubbling for $30 \mathrm{~min}$ at ambient temperature. Subsequently, the quartz jar was sealed immediately after addition of a given dose of initiator VA-044 to the mixture, and then continuously sonicated at $35-45{ }^{\circ} \mathrm{C}$ for $60-$ $90 \mathrm{~min}$. When the reaction was over, the copolymer was aged for $4 \mathrm{~h}$ at room temperature to increase the polymerization degree. Finally, the copolymer was purified by acetone and ethanol for several times and dried in a vacuum oven at $60{ }^{\circ} \mathrm{C}$ until

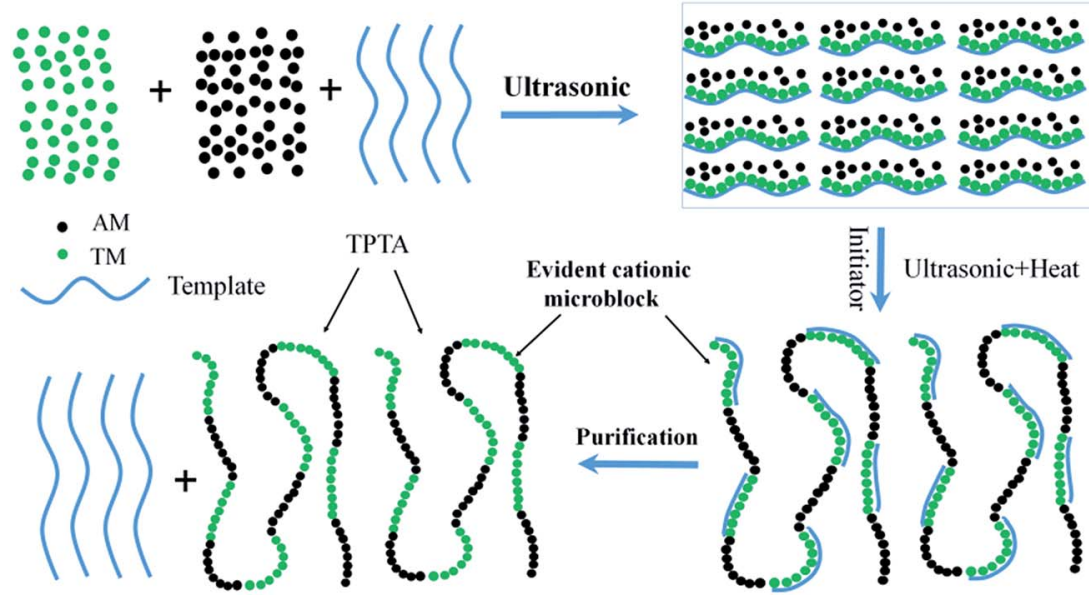

Fig. 1 The ultrasonic-initiated template copolymerization process for TPTA. 
a constant weight was obtained. The preparation of CPTA was similar to that of TPTA except that no template and ultrasonic were used. The proposed reaction routes for TPTA and CPTA were outlined in ESI Fig. S1. $\dagger$

\subsection{Characteristics of copolymers}

The intrinsic viscosities of polymers were recorded on an Ubbelohde viscosity meter (Shanghai Shenyi Glass Instrumental Co. Ltd., China) at $30{ }^{\circ} \mathrm{C}$ in a $2 \mathrm{~mol} \mathrm{~L}^{-1}$ aqueous $\mathrm{NaCl}$ solution. ${ }^{15}$ The molecular weight was calculated by Huggins equation illustrated in ESI Text S1. $\dagger$ The degree of cationic (DC) of the copolymer was measured by colloid titration method. ${ }^{\mathbf{1 6}}$ The FT-IR and ${ }^{1} \mathrm{H}\left({ }^{13} \mathrm{C}\right) \mathrm{NMR}$ of the products were recorded on a 550 Series II infrared spectrometer (Mettler Toledo Instruments Co., Ltd., Switzerland) using $\mathrm{KBr}$ pellets and an Avance 500 nuclear magnetic resonance spectrometer (Bruker Company, Ettlingen, Germany) with deuterium oxide $\left(\mathrm{D}_{2} \mathrm{O}\right)$ as the solvent, respectively. Furthermore, TGA and differential scanning calorimetry (DSC) of the polymers were carried out at a heating rate of $10{ }^{\circ} \mathrm{C} \mathrm{min}{ }^{-1}$ under a nitrogen flow of $20 \mathrm{~mL}$ $\min ^{-1}$ from 20 to $600{ }^{\circ} \mathrm{C}$ on a DTG-60H synchronal thermal analyzer (Shimadzu, Kyoto, Japan).

\subsection{Determination of $K_{M}$ and reaction kinetics}

In order to deeply comprehend the template polymerization mechanism and the effect of template on the polymerization reaction system, the association constant $\left(K_{\mathrm{M}}\right)$ and reaction kinetics were systematically investigated. The $K_{\mathrm{M}}$ (between TM and NaPAA) was measured by bag filter method. A predetermined dose of template NaPAA with a molecular weight $\left(M_{\mathrm{w}}\right)$ of 4200 was added into dialysis bag (Intercepted, $M_{\mathrm{w}} \mathrm{CO}$ 3500, MD 25-3.5, USA), and then the dialysis bag was submerged in deionized water for dialysis. During the process of dialysis, the low $M_{\mathrm{w}}$ NaPAA was removed and the remaining NaPAA became more similar and uniform. After $48 \mathrm{~h}$ dialysis, the NaPAA in the dialysis bag was precipitated by ethanol and the precipitation was dry in a vacuum oven at $60{ }^{\circ} \mathrm{C}$ for $24 \mathrm{~h}$. A certain dose of TM and the precipitation NaPAA were dissolved in deionized water in a $250 \mathrm{~mL}$ glass beaker, and then the solution $\mathrm{pH}$ was adjusted to 3.0 by $0.5 \mathrm{~mol} \mathrm{~L}^{-1} \mathrm{HCl}$ and $\mathrm{NaOH}$. Subsequently, the glass beaker was sealed immediately and kept for $24 \mathrm{~h}$ at room temperature to arrive a penetration balance. Finally, the $K_{\mathrm{M}}$ was calculated by the following formula:

$$
K_{\mathrm{M}}=\frac{[\mathrm{NaPAA} * * * * \mathrm{TM}]}{[\mathrm{NaPAA}]_{\mathrm{f}}[\mathrm{TM}]_{\mathrm{f}}}
$$

where $\left[\mathrm{NaPAA}^{* * * * \mathrm{TM}]}\right.$ was the concentration of the association of NaPAA and TM, $[\mathrm{NaPAA}]_{\mathrm{f}}$ and $[\mathrm{TM}]_{\mathrm{f}}$ were the free concentration when the dialysis kept balance. Meanwhile, the free concentration of NaPAA and TM was measured by conductometric titration method. Prior to the determination of polymerization rate $\left(R_{\mathrm{p}}\right)$, the monomer conversion rate was controlled to less than $10 \%$ by shortening the sonication time at $35^{\circ} \mathrm{C}$, and the calculation equation of $R_{\mathrm{p}}$ was described as follows:

$$
R_{\mathrm{p}}=k \times[\mathrm{M}]
$$

where $[\mathrm{M}]$ was the initial concentration of monomer, and $k$ was the slope of the $Y_{t}=\ln \left[1 /\left(1-C_{t}\right)\right]$ plot (a fitting straight line), where $C_{t}$ is the monomer conversion $(C)$ with a given reaction time at $2 \mathrm{~min}, 4 \mathrm{~min}, 6 \mathrm{~min}, 8 \mathrm{~min}$ and $10 \mathrm{~min}$, and it was determined by gravimetric method. ${ }^{17}$

\subsection{Dewatering experiment}

In this dewatering experiment, it was more reasonable and receivable to use the flocculants with the same intrinsic viscosity and cationic degree for comparison analyses. The flocculants used for the dewatering tests were listed in ESI Table 1. $\dagger$ Raw textile-dyeing sludge used in this experiment was collected from Junzhi textile-dyeing wastewater treatment plant (Chongqing, China) with a cyclic-activated sludge system. The sludge collected from this plant featured by a $\mathrm{pH}$ of 7.2, a moisture content of $99.4 \%$, a zeta potential of $-21.8 \mathrm{mV}$, a mass density of $0.946 \mathrm{~kg} \mathrm{~L}^{-1}$ and a VSS/TSS of 0.77 . A programcontrolled Jar-test apparatus (ZR4-6, Zhongrun Water Industry Technology Development Co. Ltd., China) was used in the textile-dyeing sludge dewatering experiment at ambient temperature. About $250 \mathrm{~mL}$ textile-dyeing sludge was transferred into a $500 \mathrm{~mL}$ glass beaker, and the initial $\mathrm{pH}$ of the sludge was adjusted to the set value by $\mathrm{HCl}\left(0.1 \mathrm{~mol} \mathrm{~L}^{-1}\right)$ and $\mathrm{NaOH}\left(0.1 \mathrm{~mol} \mathrm{~L}^{-1}\right)$. A certain dosage of flocculant was quickly added to the glass beaker, followed by a rapid stirring at $200 \mathrm{rpm}$ for $30 \mathrm{~s}$ and a slow stirring at $50 \mathrm{rpm}$ for $10 \mathrm{~min}$ and then it was kept for $30 \mathrm{~min} .{ }^{\mathbf{1 8}}$ The flocculation ability of the polymers was evaluated by FCMC, SRF, settling rate, zeta potential and the flocs settling behaviors. Besides, the detail analytical methods of FCMC and SRF were described in ESI Text S2. $\dagger$ The zeta potential of supernatant was measured by a Zetasizer Nano ZS90 (Malvern Instruments Ltd., Malvern, UK). After a rapid agitation, the flocs size was recorded on a laser diffraction instrument (Mastersizer 2000, Malvern, U.K.). Each measurement was repeated in triplicate and the final results were the average of three runs.

The sludge settling performance was carried out on a 1000 $\mathrm{mL}$ graduated cylinder with a total height of $29.5 \mathrm{~cm}$. After a predetermined dose of flocculant was added to the sludge sample, the graduated cylinder was immediately sealed and inverted for 4 times. Then, the sludge precipitated for $60 \mathrm{~min}$ without disturbance. The height of the sludge-liquid interface with time was immediately recorded until the height kept constant. The settling rate of the sludge conditioned by flocculant was calculated in term of the height of the sludge-liquid interface as a function of settling time in the first $150 \mathrm{~s}$. When the height of the sludge-liquid interface kept constant, a camera (Millet five, China) was used to observe the flocs morphology of conditioned sludge.

\section{Results and discussion}

\subsection{The association constant $\left(K_{M}\right)$ and reaction mechanism}

In the template polymerization system, the cationic monomer TM was absorbed and arranged along with the chain of the anionic template NaPAA under the electrostatic force, whereas 
neutral AM lagged behind TM in the competition of capturing template. Therefore, the interaction force between TM and NaPAA could be measured in term of the association constant $\left(K_{\mathrm{M}}\right)$ and the corresponding mechanism could be obtained. ${ }^{19}$ The dialysis result showed a $K_{\mathrm{M}}$ value of 12.31 when the molar ratio of TM to NaPAA was $1: 1$, which meant about three quarters of the monomer TM was absorbed by template to form a novel block structure. Based on the above analysis, the template polymerization belonged to I (ZIP) mechanism shown in Fig. 2. Furthermore, it also indicated that the NaPAA was suitable for the template polymerization.

\subsection{Effect of mole ratio of NaPAA to TM on $\boldsymbol{R}_{\mathrm{p}}$}

As one important part in the template polymerization system, NaPAA played a crucial role in the cationic monomer assembling. In the process of template polymerization, the influence of template on the conversion $(C)$ and polymerization rate $\left(R_{\mathrm{p}}\right)$ was investigated. In all cases, the TM concentration was kept constant. As shown in Fig. 3, the $C$ and $R_{\mathrm{p}}$ reached the maximum value of approximately $9.44 \%$ and $0.103 \mathrm{~mol} \mathrm{~L}^{-1}$ $\min ^{-1}$ at a NaPAA/TM molar ratio of 1 , respectively. The changing tendency of $C$ and $R_{\mathrm{p}}$ conformed to the I (ZIP) template polymerization mechanism. When the NaPAA/TM molar ratio was lower than 1, TM was adsorbed completely on the template molecular chain and it tended to be homopolymerized, and thereby the $R_{\mathrm{p}}$ increased. However, it became the inverse at a high molar ratio (NaPAA/TM $>1$ ), and the further increase in the amount of template leaded to a low filling degree of NaPAA by TM and a large molecular gap among TM, thus resulting in a decline of $R_{\mathrm{p}} \cdot{ }^{20}$ Besides, the I (ZIP) mechanism also indicated that the AM had little impact on the interaction of template NaPAA and TM in the process of template polymerization.

\subsection{Characterization of flocculants}

3.3.1. FTIR spectral analysis. Fig. 4 showed the FT-IR spectra of CPTA and TPTA. The FT-IR spectra of CPTA were almost the same with that of TPTA, except for a slight shift in the peak area. The adsorption peaks at 3444 and $1665 \mathrm{~cm}^{-1}$ originated from the strong stretching vibration of $-\mathrm{NH}_{2}$ and $\mathrm{C}=\mathrm{O}$ in the AM chain, respectively. The asymmetric stretching vibration was observed at $2942 \mathrm{~cm}^{-1}$ for $-\mathrm{CH}_{3}$ and $2845 \mathrm{~cm}^{-1}$ for $-\mathrm{CH}_{2}{ }^{21}$ The bending vibration at $1451 \mathrm{~cm}^{-1}$ was attributed $-\mathrm{CH}_{2}-$ in the $-\mathrm{CH}_{2}-\mathrm{N}^{+}$group of TM. The bending vibration at $963 \mathrm{~cm}^{-1}$ was for $-\mathrm{N}^{+}-\left(\mathrm{CH}_{3}\right)_{3}$ in TM; the characteristic chemical groups of AM and TM were all observed in CPTA and TPTA, thus indicating that CPTA and TPTA were successfully synthesized through copolymerization of AM and TM. Furthermore, the FTIR spectra results indicated that UTP did not exert influence on the functional group structure of TPTA.

3.3.2. ${ }^{1}$ H NMR spectral analysis. As an effective method, the ${ }^{1} \mathrm{H}$ NMR was always used to identify the molecular structure

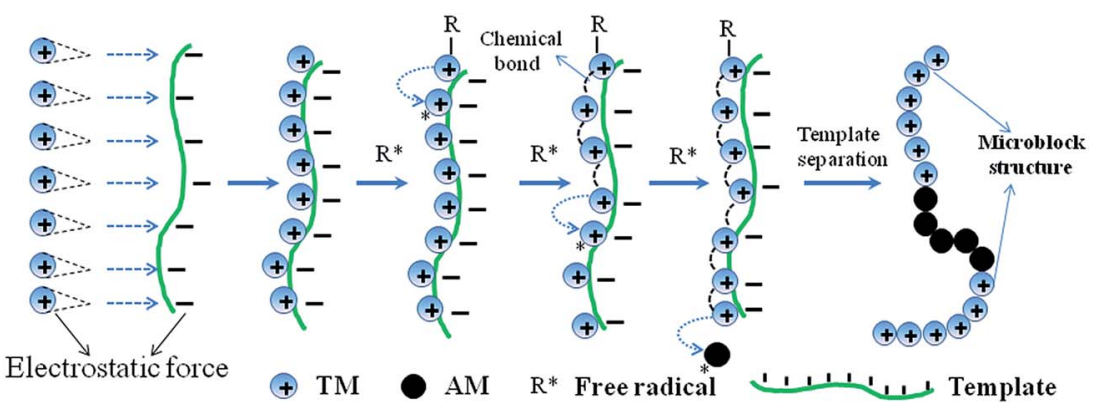

Fig. 2 The mechanism of ultrasonic-template copolymerization.
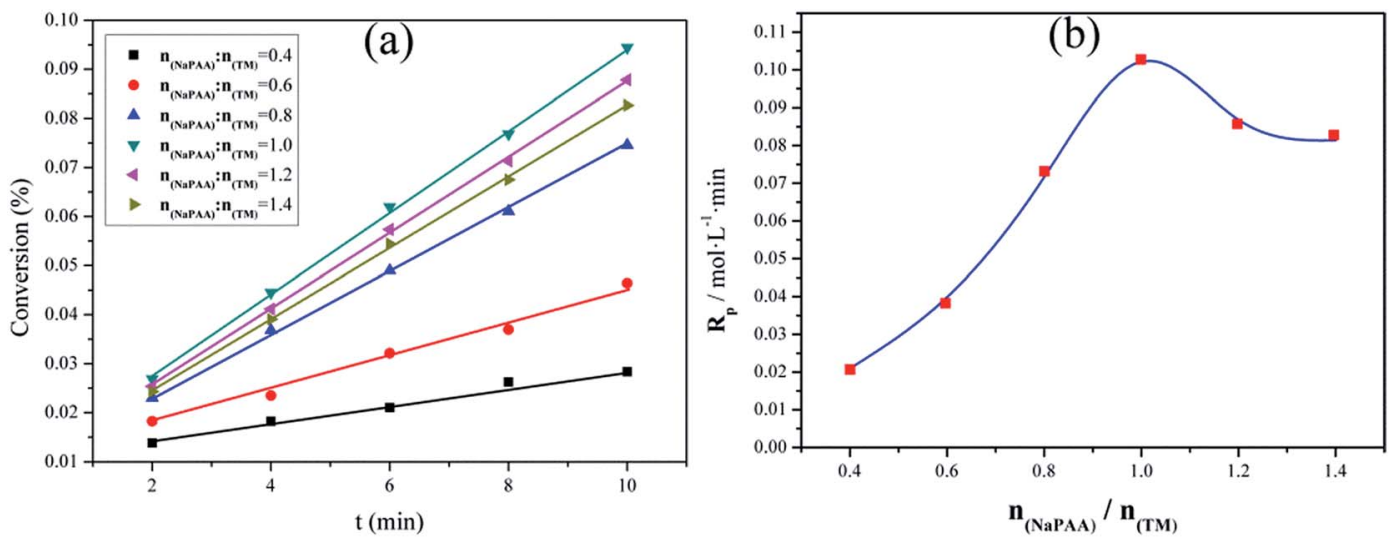

Fig. 3 Effect of mole ratio of NaPAA and TM on conversion (a) and $R_{\mathrm{p}}$ (b) at $n_{\mathrm{AM}}: n_{\mathrm{TM}}=3.5: 1, \mathrm{pH}=3.0, \mathrm{C}=35^{\circ} \mathrm{C}$ and $\mathrm{C}_{\mathrm{TM}}=5.5 \mathrm{~mol} \mathrm{~L}^{-1}$. 


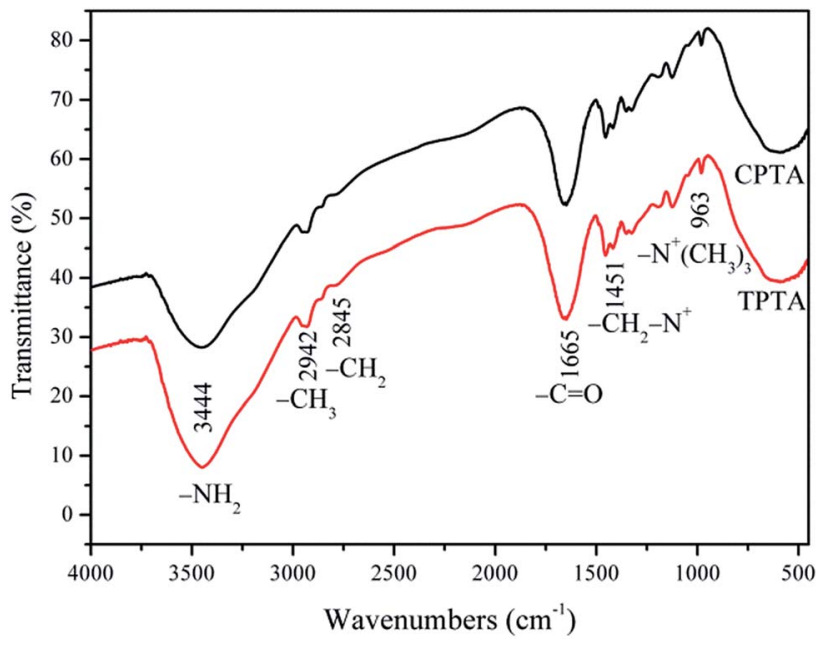

Fig. 4 FT-IR spectra of CPTA and TPTA.

in the domain of polymer science. To investigate the impact of template copolymerization on the polymer microstructure, the ${ }^{1} \mathrm{H}$ NMR of CPTA, TPTA and PAM were compared and the results were displayed in Fig. 5. It revealed that CPTA and TPTA displayed many similarities, although several subtle differences should not be ignored. The absorption peaks of protons in the $-\mathrm{CH}_{2}-\left(\mathrm{a}_{1}\right)$ and $-\mathrm{CH}-\left(\mathrm{b}_{1}\right)$ groups of both CPTA and TPTA were observed at $\delta=1.71 \mathrm{ppm}$ and $\delta=2.28 \mathrm{ppm}$, respectively, whereas those for PAM shifted. For example, the peaks shifted to $\delta=1.62 \mathrm{ppm}$ for $-\mathrm{CH}_{2}-$ (a) and $\delta=2.19 \mathrm{ppm}$ for $-\mathrm{CH}-(\mathrm{b})$ in PAM. The above disparities were caused by the different chemical environment of the protons in the polymers chain. When the copolymer of AM and TM, namely, CPTA or TPTA, was successfully synthesized, the chemical environment of the protons in the $-\mathrm{CH}_{2}-$ and $-\mathrm{CH}-$ of CPTA or TPTA was not

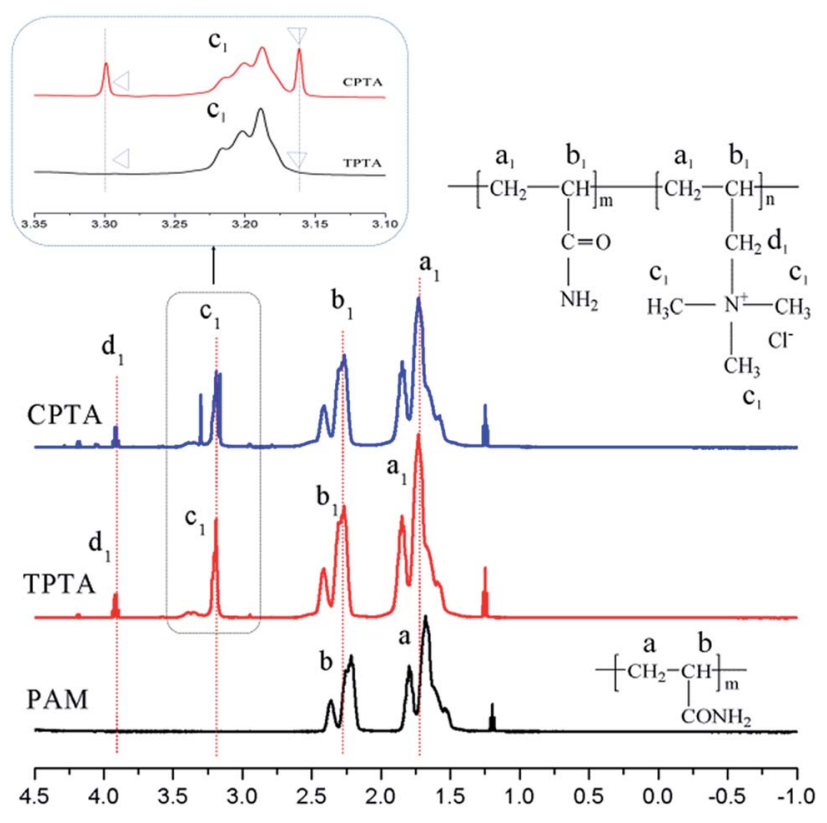

Fig. $5{ }^{1} \mathrm{H}$ NMR spectrum of the polymers. consistent with that of PAM, thus chemical shifts were not identical. ${ }^{22}$ Meanwhile, the peak of protons at $\delta=3.19 \mathrm{ppm}$ was for the three equivalent methyl groups $\mathrm{N}^{+}\left(\mathrm{CH}_{3}\right)_{3}\left(\mathrm{c}_{1}\right)$ in TM and that at $\delta=3.92 \mathrm{ppm}$ was attributed to the methylene group $\left(\mathrm{d}_{1}\right)$ which was next to the quaternary ammonium group $\left(-\mathrm{N}^{+}-\right.$ $\left.\left(\mathrm{CH}_{3}\right)_{3}\right)$ in TM. Based on the ${ }^{1} \mathrm{H}$ NMR analysis results, it indicated that CPTA and TPTA were the copolymer of AM and TM.

However, the subtle difference between CPTA and TPTA should be paid more attention and it was vital to this study. Two peaks at $\delta=3.30 \mathrm{ppm}$ and $\delta=3.16 \mathrm{ppm}$ were observed in the ${ }^{1} \mathrm{H}$ NMR spectral of CPTA shown in the rectangle in Fig. 5, whereas those in TPTA disappeared. This interesting phenomenon should be reasoned as follows. In TPTA, the cationic microblock structure was predominantly syndiotactic because of the steric and electrostatic repulsion of the pendant groups. Thus, the protons of $\mathrm{N}^{+}\left(\mathrm{CH}_{3}\right)_{3}$ in $\mathrm{TM}$ microblock structure were identical in principle and only one proton peak was observed for each group. ${ }^{23}$ By contrast, the random distribution of cationic monomer in CPTA resulted in a different chemical environment rather than that of TPTA and the protons became different with the adjacent protons and thereby two individual split protons peaks shown in a rectangle in Fig. 5 were observed for $\mathrm{N}^{+}\left(\mathrm{CH}_{3}\right)_{3}$ group. This finding was similar to the previous research. ${ }^{24}$ Therefore, it could be concluded that the cationic microblock structure in TPTA was successfully synthesized by ultrasonicinitiated template copolymerization.

3.3.3. ${ }^{13} \mathrm{C}$ NMR spectral analysis. The ${ }^{13} \mathrm{C}$ NMR of the TPTA, CPTA and PAM were also investigated, and the related peaks showed in Fig. 6. It was more evident that TPTA and CPTA showed similarities in ${ }^{13} \mathrm{C}$ NMR spectral. The resonance peaks at $34.72,42.08$ and $179.48 \mathrm{ppm}$ were ascribed to the carbon of $-\mathrm{CH}_{2}-\left(\mathrm{a}, \mathrm{a}_{1}\right),-\mathrm{CH}-\left(\mathrm{b}, \mathrm{b}_{1}\right)$ and $-\mathrm{C}=\mathrm{O}-\left(\mathrm{c}, \mathrm{c}_{1}\right)$ in the polymers (TPTA, CPTA and PAM), respectively. Meanwhile, the carbon peaks of TM were all was observed in both TPTA and CPTA. For example, the carbon peak at $53.85 \mathrm{ppm}$ was for $-\mathrm{N}^{+}\left(\mathrm{CH}_{3}\right)_{3}$ (d) and that at $66.87 \mathrm{ppm}$ was for $-\mathrm{CH}_{2}-\mathrm{N}^{+}-(\mathrm{e})$. The results of the ${ }^{13} \mathrm{C}$ NMR indicated that both $\mathrm{AM}$ and $\mathrm{TM}$ reacted and the copolymer TPTA and CPTA were formed.

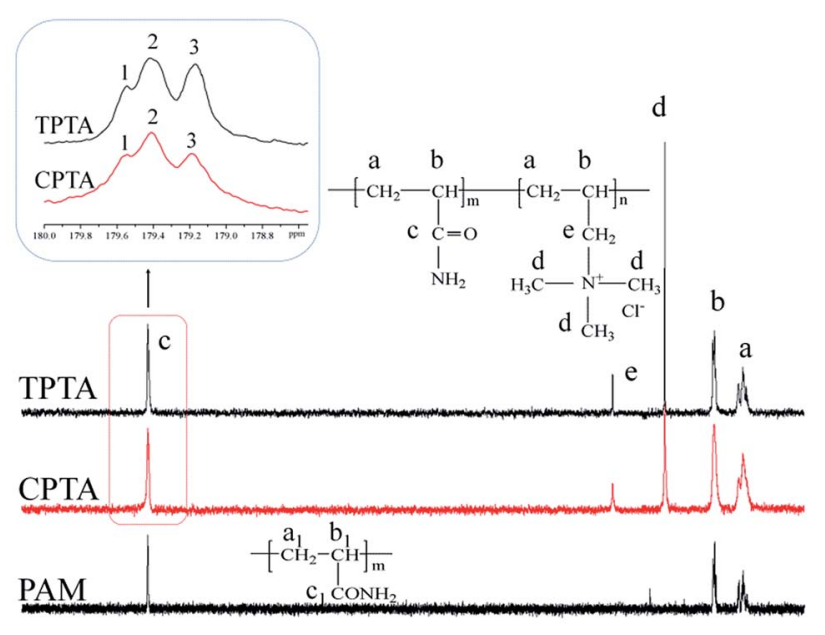

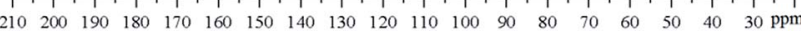
Fig. $6{ }^{13} \mathrm{C}$ NMR spectrum of the polymers. 
The microstructure of the polymer was significantly changed due to the formation of the microblock structure. The distribution of AM and TM become more ordered in TPTA rather than random, and which would increase the proportion of the AM and TM chain sequence segment. It has reported that the trials distribution and proportion could be determined by the number and relative intensity of the carbonyl carbon resonance peaks in AM, and therefore the proportion of the AM chain sequence segment in TPTA could be calculated from the area of the relative resonance peaks using the MestReNova software. ${ }^{25}$ As shown in the rectangle in Fig. 6, the peaks of 1,2 and 3 represented the AM-centered trials and were TAT, AAT and AAA, respectively. Comparing with CPAT, the peak area of AAA and TAT in TPAT shown in Table 1 increased to $47.1 \%$ and $9.2 \%$, respectively, whereas that of AAT declined to $43.7 \%$. The addition of the template generated a microblock structure (AM blocks and TM blocks) in TPAT, thereby increasing the proportion of AM chain sequence segment. Meanwhile, the proportion of TAT in TPTA was larger than that in CPTA. An explanation could be that template method could increase the monomer reaction rate and the conversion, and which was consistent with the analysis of $R_{\mathrm{p}}{ }^{22}$

3.3.4. Thermogravimetric analysis. The TGA curves of CPTA and TPTA were displayed in Fig. 7(a and b). It was obvious that three stages of the thermal decomposition were for both CPTA and TPTA, corresponding to their weight loss. The first stage occurred in the range of $30-205{ }^{\circ} \mathrm{C}$ with a mass loss of 8.2 $\mathrm{wt} \%$ for CPTA and $9.6 \mathrm{wt} \%$ for TPTA, which could be assigned to the evaporation of intramolecular and intermolecular moisture in the polymers. ${ }^{26}$ The second stage occurred in the range of 205-345 ${ }^{\circ} \mathrm{C}$ with a mass loss of $17.1 \mathrm{wt} \%$ for CPTA and $20.3 \mathrm{wt} \%$

Table 1 Relative intensity of the trials distribution of distribution acrylamide (AM) ${ }^{a}$

\begin{tabular}{llll}
\hline Copolymer & AAA $(\%)$ & AAT $(\%)$ & TAT $(\%)$ \\
\hline CPAT & 34.4 & 59.9 & 5.7 \\
TPAT & 47.1 & 43.7 & 9.2
\end{tabular}

${ }^{a}$ A, AM unit; T, TM unit; 1, TAT; 2, AAT; and 3, AAA. for TPTA, which was attributed to the imine reaction of the amide group and the thermal decomposition of methyl in the quaternary ammonium groups. And the final stage occurred beyond $345{ }^{\circ} \mathrm{C}$ with a mass loss of $44.3 \mathrm{wt} \%$ for CPTA and 36.1 wt $\%$ for TPTA, which was due to the carbonization of the copolymer. Meanwhile, two evident heat adsorption peaks were observed at 354.6 and $414.8{ }^{\circ} \mathrm{C}$ in the DSC curve of TPTA, whereas only a single peak appeared at $342.3{ }^{\circ} \mathrm{C}$ in that of CPTA. The thermal gravimetric curves of the polymers were related to their structure. The evident microblock structure, namely, the TM and AM block was generated by template copolymerization, and therefore two characteristic peaks of heat adsorption were observed for TPTA in the second stage. However, the monomer in CPTA distributed randomly rather than orderly and no blocky structure formed, and therefore just a single adsorption peak was appeared. The previous researches have reported the similar findings., ${ }^{9,27}$ Moreover, the TGA results also indicated that both TPTA and CPTA had inferior thermal stabilities.

\subsection{Dewatering test}

3.4.1. Effect of the dosage on sludge dewatering performance. The effect of flocculants dosage on sludge flocculation performance were investigated in terms of FCMC, SRF and zeta potential. As showed in Fig. 8, the FCMC and SRF decreased with an increase in dose, and then represented a gradually upward trend. It was quite clear that TPTA showed a better flocculation performance than the other there in the full dose range, and their optimum dosages were all at $40 \mathrm{mg} \mathrm{L}^{-1}$. In this condition, the sludge dewatering performance reached the best, and their FCMC and SRF values were both in the following order: TPTA (FCMC: $73.3 \%$; SRF: $5.0 \times 10^{12} \mathrm{~m} \mathrm{~kg}^{-1}$ ) < CPTA (FCMC: $74.2 \%$; SRF: $5.2 \times 10^{12} \mathrm{~m} \mathrm{~kg}^{-1}$ ) < CCPDA (FCMC: 75.8\%; SRF: $5.5 \times 10^{12} \mathrm{~m} \mathrm{~kg}^{-1}$ ) < CCPMA (FCMC: 76.9\%; SRF: $\left.5.9 \times 10^{12} \mathrm{~m} \mathrm{~kg}^{-1}\right)$. However, too large flocculant dosage (>40 $\mathrm{mg} \mathrm{L}^{-1}$ ) leaded to a deterioration in both FCMC and SRF, which was caused by the increased charge repulsion between the sludge colloidal particles and the subsequent stabilization of those particles.

The zeta potential of the sludge colloid system was investigated to comprehend the flocculation mechanism and explain the dewatering ability discrepancy among the polymers. In
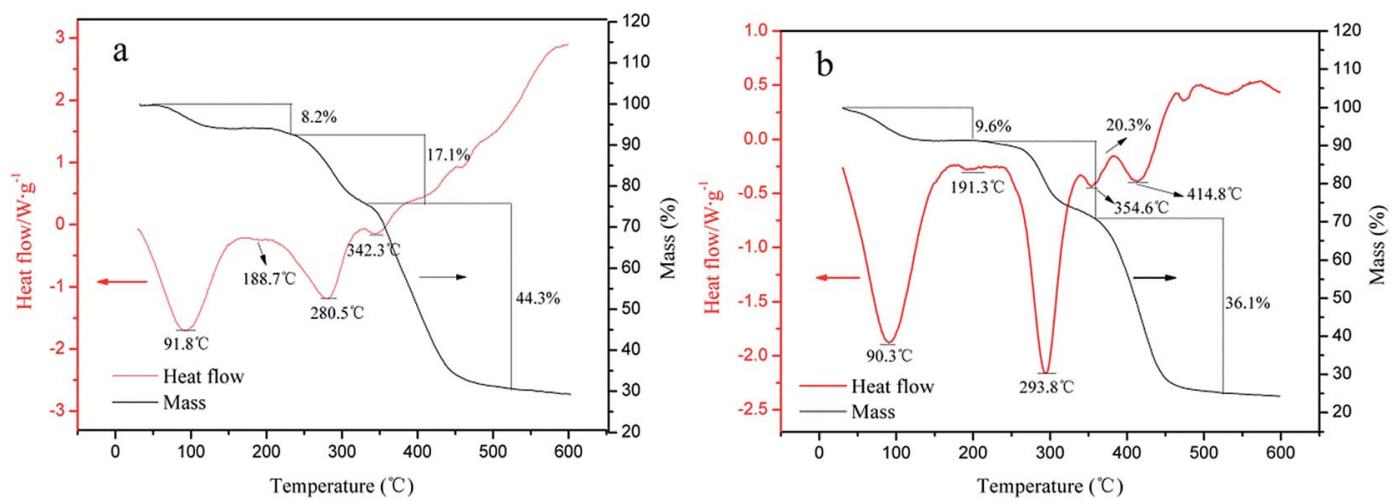

Fig. 7 Thermogravimetric curves of (a) CPTA and (b) TPTA. 

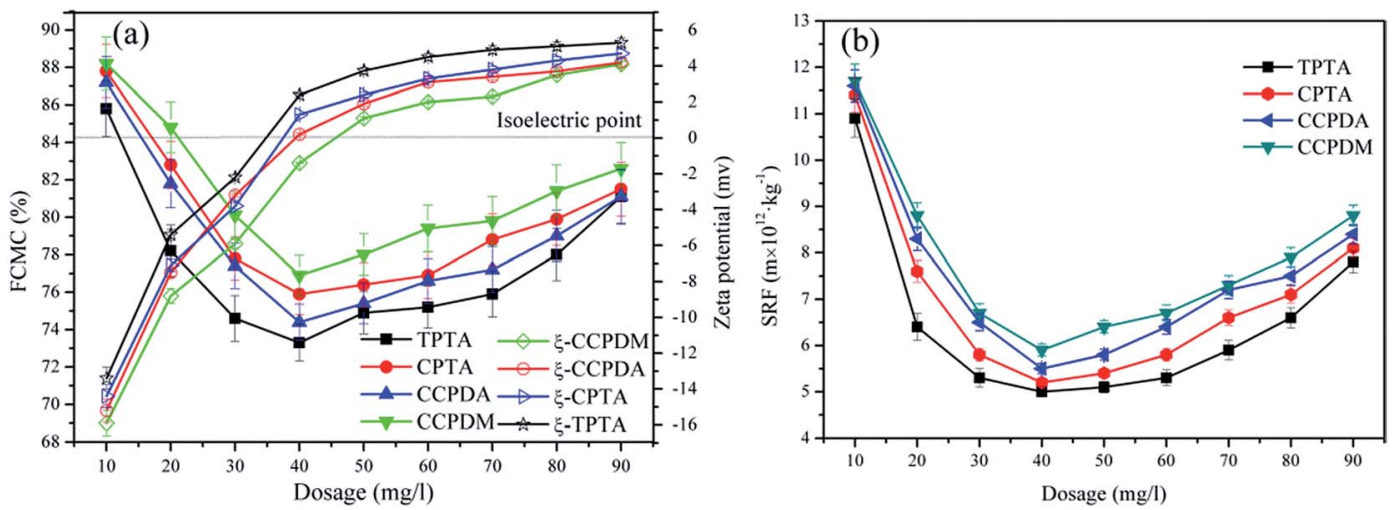

Fig. 8 Effect of the dosage on (a) FCMC and zeta potential, and (b) SRF.

Fig. 8, the zeta potential of the supernatant conditioned by TPTA, CPTA, CCPDA and CCPMA displayed a similar trend, that was, it increased with the increasing polymer dosage, but that flocculated by TPTA increased the fastest. Comparing with CPTA, CCPDA and CCPMA, the cationic microblocks in TPTA could greatly enhance the charge neutralization ability, and therefore the zeta potential increased to the isoelectric point at a lower dosage for TPTA $\left(34.9 \mathrm{mg} \mathrm{L}^{-1}\right)$ than those of CPTA (37.3 $\left.\mathrm{mg} \mathrm{L}^{-1}\right)$, CCPDA (39.7 $\mathrm{mg} \mathrm{L}^{-1}$ ) and CCPMA (45.8 $\mathrm{mg} \mathrm{L}^{-1}$ ). Charge neutralization played a vital role in the sludge flocculation because the negative charged colloidal could be neutralized and destabilized by cationic flocculant to form large flocs. ${ }^{28}$ The cationic microblocks of TPTA not only had a strong charge neutralization ability but also strengthened the stretch of molecular chain by its strong charge repulsion which was favorable for bridging, thus increasing the flocculation efficiency. As a result, TPTA showed the best flocculation performance among them in terms of FCMC and SRF. Besides, the optimum dosage was not consistent with that at the isoelectric point, which indicated that adsorption and bridging effects also made a contribution to the sludge dewatering.

3.4.2. Effect of $\mathbf{p H}$ on sludge dewatering performance. Fig. 9 displayed the impact of the $\mathrm{pH}$ on the FCMC, zeta potential and SRF at the dosage of $40 \mathrm{mg} \mathrm{L}^{-1}$ and the intrinsic viscosity of $2.3 \mathrm{dL} \mathrm{g}^{-1}$. When the $\mathrm{pH}$ increased from 2.0 to 11.0 ,
FCMC and SRF showed a similar trend of decreasing initially and then increasing, whereas zeta potential revealed a declining trend. The sludge dewatering performances of polymers were greatly affected by $\mathrm{pH}$, which indicated that $\mathrm{pH}$ played an important role in the sludge flocculation process. The strong acid (pH: 2.0-4.0) and alkali (pH: 9.0-11.0) conditions enhanced the charge intensity on colloidal particles surface and resulted in a strong repulsive force between the particles. ${ }^{29}$ Consequently, the adsorbed particles were separated from the polymers chain and the sludge dewatering performance became less than satisfactory. Comparing with CPTA, CCPDA and CCPMA, TPTA showed the highest dewatering efficiency among them in the full $\mathrm{pH}$ range, and its minimum SRF of $4.9 \times 10^{12} \mathrm{~m} \mathrm{~kg}^{-1}$ and FCMC of $72.1 \%$ were acquired at $\mathrm{pH}$ 7.0. Meanwhile, the zeta potential of the conditioned sludge system was the maximum for TPTA, compared with the other three, and which indicated that the cationic microblock structure could strengthen the charge neutralization ability. In addition, the cationic microblock structure was also favorable for compressing the thickness of the electric double layer. The strong positive charge repulsive force of the cationic microblock would effectively compress the diffusion layer of negative charged particles and reduce its thickness, and therefore the charge repulsion of the negative charged particles decreased. Furthermore, TPTA was
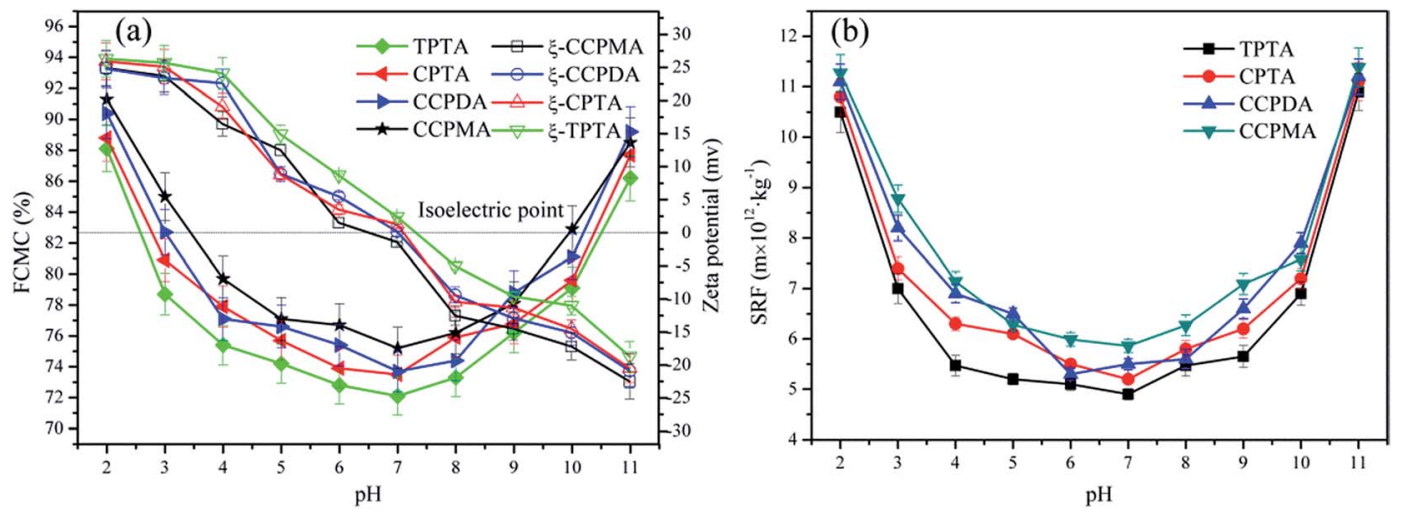

Fig. 9 Effect of the pH on the (a) FCMC and zeta potential, and (b) SRF. 
demonstrated to have an excellent sludge dewatering performance in a broad scope of $\mathrm{pH}$, which indicated that this novel flocculant could be widely applied in sludge dewatering in different industries such as paper mill, printing and dyeing mill and wastewater treatment plant.

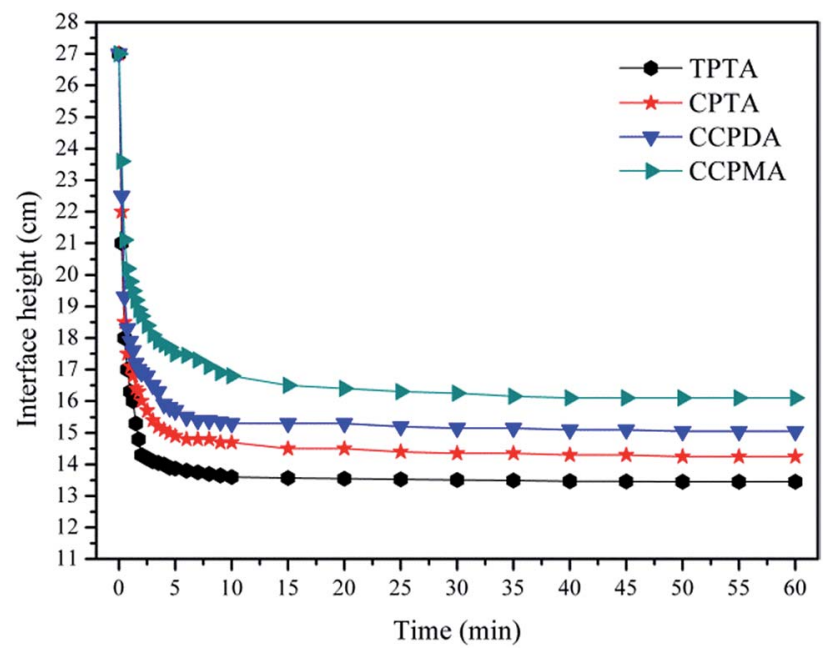

Fig. 10 Flocs interface heights of the polymers.

\subsection{Effect of flocculants on sludge settling behaviors and the mechanism}

The sludge settling behaviors has been widely used to evaluate the flocculation performance and sludge dewatering ability of the flocculants. Generally speaking, the fast settle rate and large size of flocs meant a good dewatering ability. ${ }^{30}$ In this study, the settling behaviors of the flocs conditioned with flocculants at dosage of $40 \mathrm{mg} \mathrm{L}^{-1}$ and the intrinsic viscosity of $2.3 \mathrm{dL} \mathrm{g}^{-1}$ were investigated in terms of flocs settling rate and size distribution, and the results were shown in Fig. 10 and 11.

In Fig. 10, the ultimate sediment height and the corresponding settling rate for TPTA were the best among the flocculants, and the order was as follows: TPTA $(13.4 \mathrm{~cm} ; 5.44 \mathrm{~cm}$ $\left.\min ^{-1}\right)>$ CPTA $\left(14.2 \mathrm{~cm} ; 5.12 \mathrm{~cm} \mathrm{~min}^{-1}\right)>$ CCPDA $(15.3 \mathrm{~cm}$; $\left.4.68 \mathrm{~cm} \mathrm{~min}^{-1}\right)>$ CCPMA $\left(16.5 \mathrm{~cm} ; 4.20 \mathrm{~cm} \mathrm{~min}^{-1}\right)$. The TPTA cationic microblocks in the TPTA worked well in neutralizing the negative charged sludge particles. As long as the negative charged sludge particles became neutral, it was more likely to make the particles incorporate, and then bridging occurred and more sites of the polymer would adsorb the neutral particles. Consequently, the particles were anchored in the polymer chain and formed large and compact flocs under the effect of polymer chain strongly twisting and closely overlaying. However, the condition for CPTA was reverse. The charge neutralization
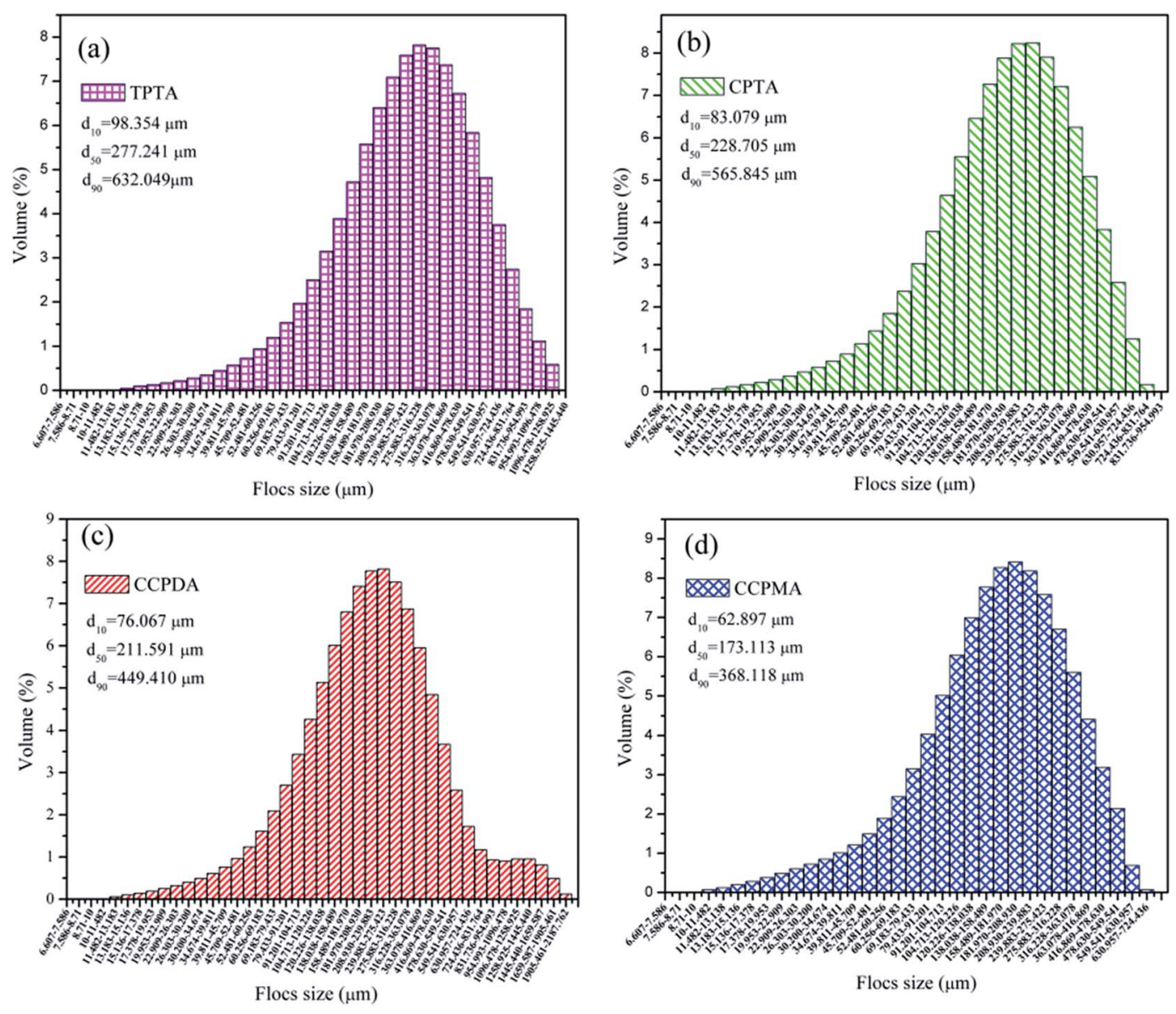

Fig. 11 Sludge flocs size distribution for (a) TPTA, (b) CPTA, (c) CCPDA and (d) CCPMA. 


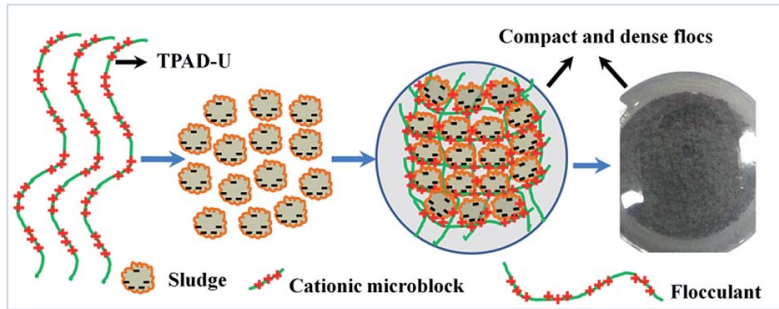

Fig. 12 Possible flocculation mechanism of the TPTA.

ability would be discounted by the random distribution of cationic monomer in CPTA molecular chain and thus the sludge could not be flocculated completely. As a result, the flocs became small and sank slow.

Comparison in flocs size distribution of the polymers was recorded. As shown in Fig. 11, the flocs size of TPTA was larger than the others, and this difference was more evident. For example, the flocs size characterized with the median equivalent volumetric diameter $\left(d_{50}\right)$ was $277.241 \mu \mathrm{m}, 228.705 \mu \mathrm{m}$, $211.591 \mu \mathrm{m}$ and $173.113 \mu \mathrm{m}$ for TPTA, CPTA, CCPDA and CCPMA, respectively. The large flocs size was attributed to the effect of the cationic microblocks in TPTA. The flocculation was mainly dominated by charge neutralization, then bridging, and which was the reason why the flocs size of TPTA was much larger than that of CPTA. Meanwhile, the sludge morphology conditioned by the TPTA and CPTA were record and the results were shown in ESI Fig. S2. $\dagger$ It was more clear that the flocs for TPTA displayed a compact, dense and beehive structure. However, that for CPTA was small and lose, and the shape of the flocs was a sand structure. Therefore, the cationic microblocks in polymer were so beneficial in improving and strengthening the sludge dewatering behaviors. According to the analyses of flocs size, flocs settle rate, flocs morphology and the zeta potential discussed in 3.4 and 3.5, the possible flocculation mechanism of the template polymer TPTA could be summarized as follows: (1) the cationic microblocks in TPTA greatly enhanced the charge neutralization and thereby the negative charge particles were neutralized completely; (2) the cationic microblocks of TPTA could generated strong charge repulsion between the molecular chain, and which was favorable for the polymer chain expansion. Therefore, the cationic microblocks could enhance bridging ability ${ }^{31}$ and (3) under the combination of charge neutralization and bridging, the large, compact and beehive-shaped flocs formed and had a fast settling rate. ${ }^{32,33}$ The possible flocculation mechanism of the TPTA was showed in Fig. 12.

\section{Conclusions}

The novel cationic microblock structure was demonstrated to be formed through the structure property analysis of TPTA. The template polymerization mechanism was investigated through the association constant $\left(K_{\mathrm{M}}\right)$ and reaction kinetics. Finally, the corresponding sludge dewatering performance of TPTA was evaluated and the possible flocculation mechanism was also discussed. The main conclusions were as follows:
(1) The template polymerization mechanism was demonstrated to be the I (ZIP) mechanism through a high $K_{\mathrm{M}}$ value $\left(K_{\mathrm{M}}: 12.31\right)$. In addition, when the mole ratio of NaPAA to TM was $1: 1$, the polymerization rate $\left(R_{\mathrm{p}}\right)$ reached the top value of $0.102 \mathrm{~mol} \mathrm{~L}^{-1} \mathrm{~min}^{-1}$, which proved the template polymerization followed the I (ZIP) mechanism again.

(2) The results of FT-IR, ${ }^{1} \mathrm{H}\left({ }^{13} \mathrm{C}\right)$ NMR and TGA indicated that the TPTA with cationic microblock structure was successfully synthesized using ultrasonic-initiated template copolymerization. In addition, the TGA results also indicated that both TPTA and CPTA had inferior thermal stabilities.

(3) Comparing with CPTA, CCPDA and CCPMA, TPTA showed an excellent dewatering performance and a wide $\mathrm{pH}$ range for application. When the optimal condition was at $\mathrm{pH}$ of 7.0, dosage of $40 \mathrm{mg} \mathrm{L}^{-1}$ and the intrinsic viscosity of $2.3 \mathrm{dL} \mathrm{g}^{-1}$, the minimum SRF of $4.9 \times 10^{12} \mathrm{~m} \mathrm{~kg}^{-1}$ and FCMC of $72.1 \%$ were acquired. Besides, the flocs conditioned with TPTA showed excellent settling behaviors, such as a rapid settling rate, a large size, and a dense structure.

(4) The cationic microblocks in the polymer played a vital role in improving and enhancing the flocculation. This novel cationic microblock structure could greatly enhanced charge neutralization ability and bridging. In this case, the negative charge particles were neutralized completely and aggregated to form large flocs, and thus an excellent flocculation performance was observed.

\section{Abbreviations}

UTP Ultrasonic-initiated template copolymerization AM Acrylamide

DAC Acryloyloxyethyl trimethyl ammonium chloride

DMC Methacryloxyethyl trimethyl ammonium chloride

NaPAA Sodium polyacrylate

MW Molecular weight

TPTA Template copolymer of TM and AM

VA-044 2,2'-Azobis[2-(2-imidazolin-2-yl)propane] dihydrochloride

CPAM Cationic polyacrylamide

$K_{\mathrm{M}} \quad$ Association constant

$R_{\mathrm{p}} \quad$ Polymerization rate

CCPDA Commercial copolymerization of AM and DAC

CCPMA Commercial copolymerization of AM and DMC

FTIR Fourier transform-infrared spectroscopy

${ }^{1} \mathrm{H}\left({ }^{13} \mathrm{C}\right) \quad{ }^{1} \mathrm{H}\left({ }^{13} \mathrm{C}\right)$ nuclear magnetic resonance spectroscopy

NMR

DSC/TGA Differential scanning calorimetry and thermogravimetric analysis

FCMC Filter cake moisture content

SRF The specific resistance to filtration

\section{Acknowledgements}

The authors are grateful for the financial support provided by the National Natural Science Foundation of China (Project No. 21677020 and 21477010). 


\section{References}

1 J. Y. Liang, X. A. Ning, T. C. An, J. Sun, Y. P. Zhang and Y. J. Wang, J. Hazard. Mater., 2016, 314, 1-10.

2 V. Jegatheesan, B. K. Pramanik, J. Chen, D. Navaratna, C. Chang and L. Shu, Bioresour. Technol., 2016, 204, 202-212.

3 Y. J. Sun, H. L. Zheng, J. Zhai, H. K. Teng, C. Zhao, C. L. Zhao and Y. Liao, PLoS One, 2014, 9, 10.

4 G. R. Chang, J. C. Liu and D. J. Lee, Water Res., 2001, 35, 786794.

5 H. L. Zheng, Y. J. Sun, C. J. Zhu, J. S. Guo, C. Zhao, Y. Liao and Q. Q. Guan, Chem. Eng. J., 2013, 234, 318-326.

6 Z. L. Yang, B. Y. Gao, C. X. Li, Q. Y. Yue and B. Liu, Chem. Eng. J., 2010, 161, 27-33.

7 K. E. Lee, N. Morad, B. T. Poh and T. T. Teng, Desalination, 2011, 270, 206-213.

8 L. H. Lu, Z. D. Pan, N. Hao and W. Q. Peng, Water Res., 2014, 57, 304-312.

9 Q. Q. Guan, H. L. Zheng, J. Zhai, C. Zhao, X. K. Zheng, X. M. Tang, W. Chen and Y. J. Sun, Ind. Eng. Chem. Res., 2014, 53, 5624-5635.

10 Y. X. Zhang, F. P. Wu, M. Z. Li and E. J. Wang, J. Phys. Chem. $B, 2005,109,22250-22255$.

11 S. Połowiński, Prog. Polym. Sci., 2002, 27, 537-577.

12 X. F. Cui, J. W. Talley, G. J. Liu and S. L. Larson, Water Res., 2011, 45, 3300-3308.

13 L. G. Qiu, Z. Q. Li, Y. Wu, W. Wang, T. Xu and X. Jiang, Chem. Commun., 2008, 3642-3644.

14 D. Gopi, J. Indira, L. Kavitha, M. Sekar and U. K. Mudali, Spectrochim. Acta, Part A, 2012, 93, 131-134.

15 H. L. Zheng, J. Y. Ma, C. J. Zhu, Z. Zhang, L. W. Liu, Y. J. Sun and X. M. Tang, Sep. Purif. Technol., 2014, 123, 35-44.
16 H. J. Ren, W. N. Chen, Y. F. Zheng and Z. K. Luan, React. Funct. Polym., 2007, 67, 601-608.

17 H. Z. Shang, J. P. Liu, Y. B. Zheng and L. G. Wang, J. Appl. Polym. Sci., 2009, 111, 1594-1599.

18 Z. Chen, W. J. Zhang, D. S. Wang, T. Ma and R. Y. Bai, Water Res., 2015, 83, 367-376.

19 X. Li, H. L. Zheng, B. Y. Gao, Y. J. Sun and B. Z. Liu, Chemosphere, 2017, 167, 71-81.

$20 \mathrm{H}$. T. van de Grampel, Y. Y. Tan and G. Challa, Macromolecules, 1991, 24, 3767-3772.

21 Y. Liao, H. L. Zheng, L. Qian, Y. J. Sun, L. Dai and W. W. Xue, Ind. Eng. Chem. Res., 2014, 53, 11193-11203.

22 Z. A. Zhang, H. L. Zheng, F. Huang, X. Li, S. Y. He and C. Zhao, Ind. Eng. Chem. Res., 2016, 55, 9819-9828.

23 Z. Abdollahi, M. Frounchi and S. Dadbin, Ind. Eng. Chem. Res., 2011, 17, 580-586.

24 W. Chen, H. L. Zheng, Q. Q. Guan, H. K. Teng, C. L. Zhao and C. Zhao, Ind. Eng. Chem. Res., 2016, 55, 2892-2902.

25 S. Zhang, G. S. Huang and J. R. Wu, J. Macromol. Sci., Part B: Phys., 2011, 50, 2203-2213.

26 C. Y. Ou, C. H. Zhang, S. D. Li, L. Yang, J. J. Dong, X. L. Mo and M. T. Zeng, Carbohydr. Polym., 2010, 82, 1284-1289.

27 J. A. Mapkar, G. Iyer and M. R. Coleman, Appl. Surf. Sci., 2009, 255, 4806-4813.

28 J. Zhou, F. W. Liu and C. Y. Pan, PLoS One, 2014, 9, 5.

29 J. C. Wei, B. Y. Gao, Q. Y. Yue, Y. Wang, W. W. Li and X. B. Zhu, Water Res., 2009, 43, 724-732.

30 Y. Q. Zhao, Sep. Purif. Technol., 2004, 35, 71-80.

31 L. H. Lu, Z. D. Pan, N. Hao and W. Q. Peng, Water Res., 2014, 57, 304-312.

32 B. Bolto and J. Gregory, Water Res., 2007, 41, 2301-2324.

33 Y. J. Sun, H. L. Zheng, J. Zhai, H. K. Teng, C. Zhao, C. L. Zhao and Y. Liao, PLoS One, 2014, 9, 10. 